Research Article

\title{
Liquid-Assisted Electrospinning Three-Dimensional Polyacrylonitrile Nanofiber Crosslinked with Chitosan
}

\author{
Xiaoli Yang, ${ }^{1}$ Xue Chen, ${ }^{1}$ Jingyi Zhao, ${ }^{1}$ Wenlu Lv, ${ }^{1}$ Qilu Wu, ${ }^{1}$ Huajian Ren $\mathbb{D}^{2}$ \\ Chuntao Chen $\left(\mathbb{0},{ }^{1}\right.$ and Dongping Sun $\mathbb{1}^{1}{ }^{1}$ \\ ${ }^{1}$ Institute of Chemicobiology and Functional Materials, Nanjing University of Science and Technology, 200 Xiao Ling Wei Street, \\ Nanjing, Jiangsu Province, China \\ ${ }^{2}$ Department of General Surgery, Jinling Hospital, Medical School of Nanjing University, 305 East Zhongshan Road, Nanjing, \\ Jiangsu Province, China
}

Correspondence should be addressed to Huajian Ren; rhj1288@163.com, Chuntao Chen; chchunt@njust.edu.cn, and Dongping Sun; sundpe301@163.com

Received 11 November 2021; Accepted 2 December 2021; Published 29 December 2021

Academic Editor: Jinjian Huang

Copyright (C) 2021 Xiaoli Yang et al. This is an open access article distributed under the Creative Commons Attribution License, which permits unrestricted use, distribution, and reproduction in any medium, provided the original work is properly cited.

Electrospinning has become a popular nanotechnology for the fabrication of tissue engineering scaffolds, which can precisely regulate fiber diameter and microstructure. Herein, we have prepared a three-dimensional polyacrylonitrile (PAN) nanofiber by liquid-assisted electrospinning. The spacing between PAN nanofibers can reach to $15-20 \mu \mathrm{m}$, as the uniform internally connected pore structure can be formed, through the regulation of parameters. Furthermore, the chitosan attached to the asprepared nanofibers gives the material antibacterial effect and increases its biocompatibility. Meanwhile, the special structure of chitosan also provides the possibility for further loading drugs in dressings in the future. This newly developed nanocomposite seems to be highly suitable for wound healing due to its unique properties of biodegradability, biocompatibility, and antimicrobial effectiveness.

\section{Introduction}

As the largest organ of the human body, the skin is the first barrier for the human body to protect itself. However, due to the physical characteristics of the skin and its associated soft tissue, it is vulnerable to external stimulation and is damaged easily. In China, the number of trauma patients seeking medical treatment is as high as 62 million times per year, among which the number of deaths is about 600-700 thousand. Therefore, there is a great clinical demand for trauma repair.

Skin dressing materials can replace the damaged skin and resist the secondary injury caused by external mechanical factors, such as the invasion and touch of foreign bodies, as well as resist the secondary pollution and injury caused by chemical factors, and prevent the wound from drying and the loss of body fluid. Traditional medical dressings mainly include cotton ball, cotton gauze and other natural fiber materials, which play the role of physical isolation. However, there are some defects, such as retearing at the scab of the wound during cleaning, easy infection of the wound, and poor hemostatic performance. Biomedical dressings can overcome those shortcomings and become the focus of researchers.

Electrospinning is an effective, versatile, and scalable technique of preparing the skin dressing materials from a variety of synthetic or natural polymers with diameters down to the nanoscale $[1,2]$. Moreover, there are many controllable parameters to control the fiber diameter, morphology, and secondary structure more accurately. In particular, the use of liquid as a receiving device for electrospinning nanofibers could obtain the interconnected 3D (three-dimensional) structure $[3,4]$. Compared with most multilayer electrospinning techniques $[5,6]$ and template techniques [7], the 3D electrospinning technique of liquid reception is more concerned with increasing fiber spacing than with the construction of macroscopic 3D structure [8-10]. And compared 


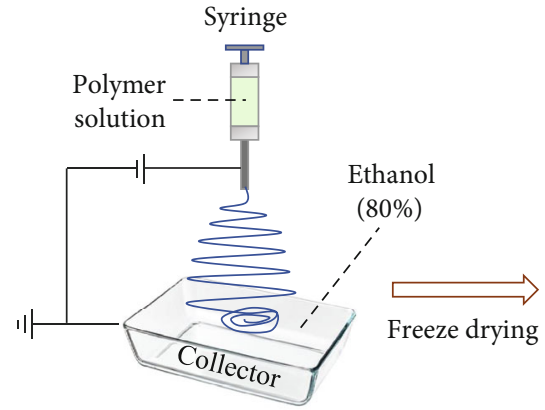

Electrospinning

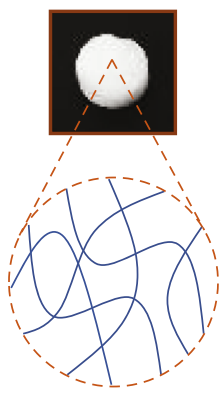

FNFSs

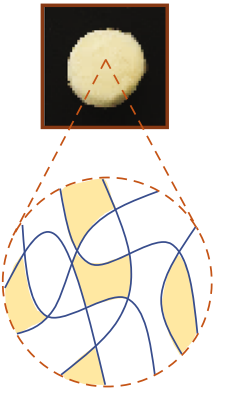

CNSs

Scheme 1: Schematic illustration of the setup for liquid-assisted electrospinning.

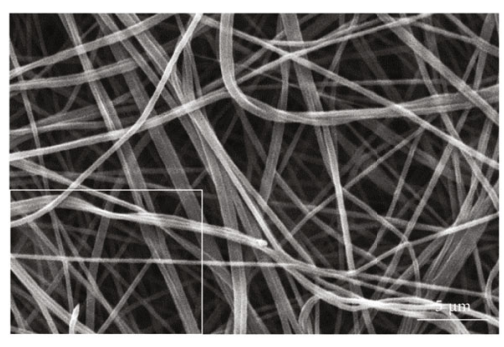

(a)

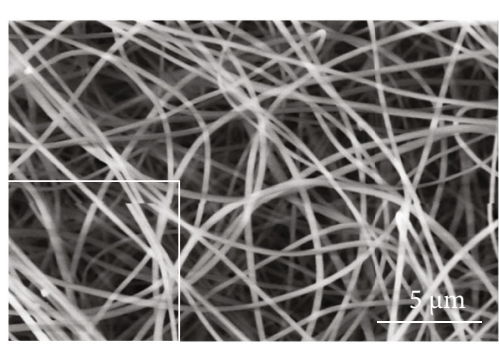

(c)

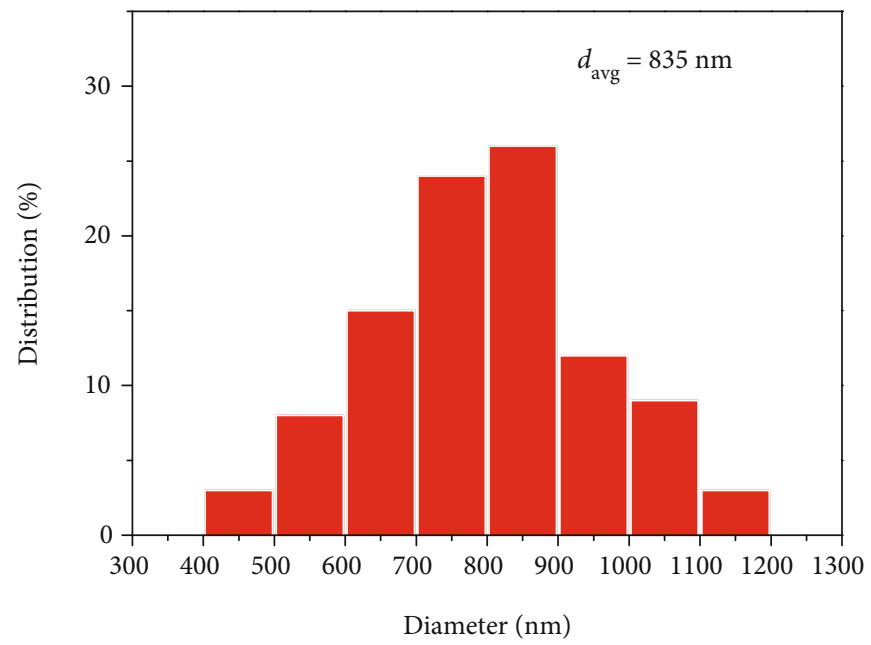

(b)

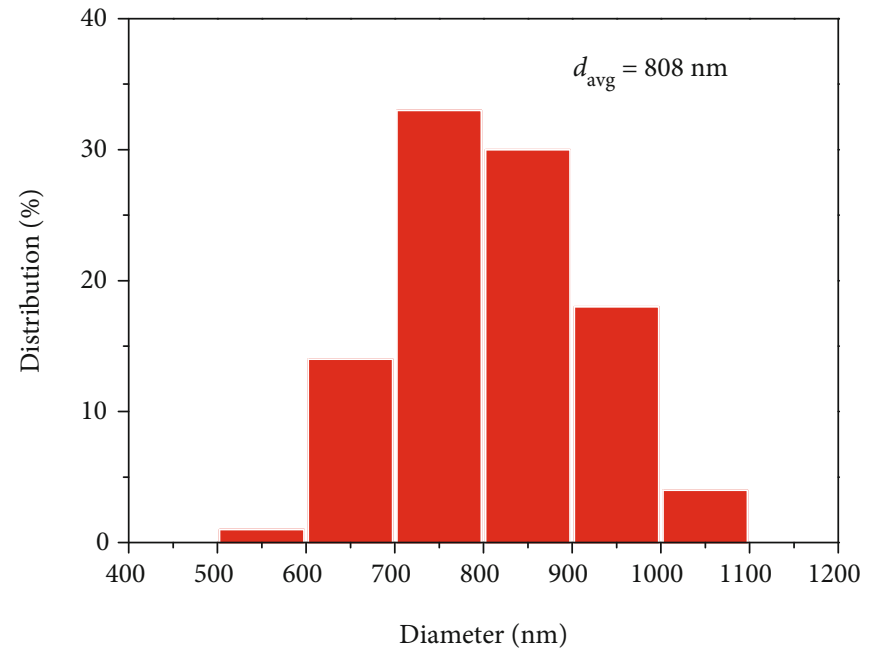

(d)

Figure 1: (a) SEM images and (b) fiber diameter distribution of electrospinning 2D-PAN fiber mats; (c) SEM images and (d) fiber diameter distribution of the electrospinning 3D-FNFS nanofiber.

with the pore-forming agent technique [11], the aperture is not too large and the pore distribution is more uniform [3]. In addition, this technique is convenient to operate and does not need other complex postprocessing technology such as salt leaching or template removal [12].
Polyacrylonitrile (PAN) is a kind of semicrystalline synthetic organic polymer, which is an important raw material of the ultrafiltration membrane, reverse osmosis hollow fiber, fabric fiber, and carbon fiber [13]. Meanwhile, the cost of industrial PAN staple fibers is low while the mechanical 


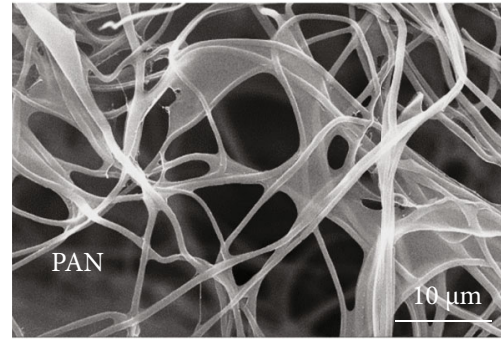

(a)

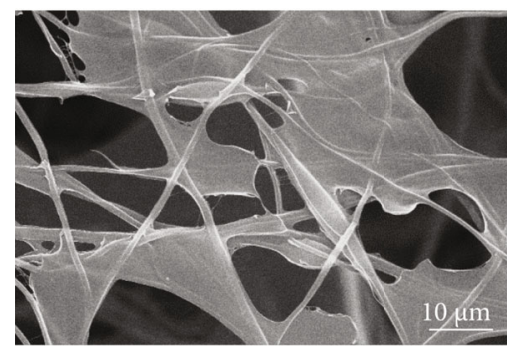

(c)

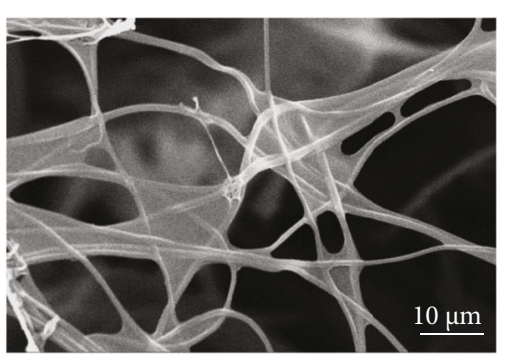

(b)

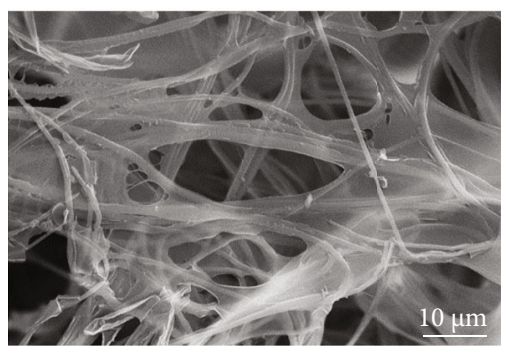

(d)

Figure 2: SEM images of (a) $\mathrm{CNS}_{0.050}$

(b) $\mathrm{CNS}_{0.075}$

(c) $\mathrm{CNS}_{0.100}$, and (d) $\mathrm{CNS}_{0.125}$ (scale bar, $10 \mu \mathrm{m}$ ) composites.

properties are stable. In addition, PAN solution in electrostatic field is easy to be spun into silk, which could provide the cells with an environment that closely resembles their native extracellular matrix (ECM). It is expected that compared with general materials, it has better cytocompatibility, which is conducive to promoting the reproductive growth of wound cells and accelerating wound healing. In addition, 3D structure provides more crosslinking sites, which is conducive to the adhesion of materials and the adsorption of tissue fluid [14-16]. Consequently, electrospinning nanofibers are considered to be an effective materials in tissue engineering application, which is of great significance in real life [17-20]. As a biomaterial with rich pore structure, electrospinning nanofibers can be used as scaffold for three-dimensional cell culture in tissue engineering [21]. Moreover, due to their porous structure which provides sufficient space for cells' uniform distribution and facile delivery of oxygen and nutrients, the 3D nanofiber can mimic the topology and biological functions of the extracellular matrix (ECM) for guided cell growth.

In this study, we report the preparation of 3D PAN nanofiber scaffolds by electrospinning and crosslinking with different concentrations of chitosan. The 3D network structure was formed by the stacking of one-dimensional nanowires, and the amino group of chitosan was used to complete the crosslinking to enhance the antibacterial property of the material. These scaffolds were then characterized by analyzing the morphological structure and chemical structure, as well as examining the cytotoxicity and antibacterial property.

\section{Materials and Methods}

2.1. Materials. N,N-Dimethylformamide (DMF, ACS reagent), chitosan, glutaraldehyde, acetic acid, glycine, and other reagents used for the preparation of PBS buffer were purchased from Sigma-Aldrich. To prepare the PBS buffer, $8.0 \mathrm{~g} \mathrm{NaCl}, 0.2 \mathrm{~g} \mathrm{KCl}, 1.44 \mathrm{~g} \mathrm{Na}_{2} \mathrm{HPO}_{4}$, and $0.24 \mathrm{~g} \mathrm{KH}_{2} \mathrm{PO}_{4}$ were dissolved in $800 \mathrm{~mL}$ deionized water. $\mathrm{HCl}$ solution and $\mathrm{NaOH}$ solution were used to adjust the $\mathrm{pH}$ to 7.4 , and finally, the solution was diluted with deionized water to $1000 \mathrm{~mL}$. The PAN staple fibers (Macklin) were dissolved in $\mathrm{N}, \mathrm{N}$-dimethylformamide (DMF) at a concentration of $10 \%(w / v)$ and magnetic stirred at $50^{\circ} \mathrm{C}$ overnight to obtain the electrospinning precursor solution. Other cell culture media and supplements were purchased from Sigma.

2.2. Electrospinning. The solution for electrospinning was placed in a $1 \mathrm{~mL}$ syringe equipped with a 23 -gauge needle. The solution was dispersed by a syringe pump at a feeding rate of $1.0 \mathrm{~mL} / \mathrm{h}$, with a humidity of $40-53 \%$. The collector was a conductive metal square shell container covered with aluminum foil, wherein the liquid was a mixed solution of ethanol and water $(4: 1, V / V)$. The typical distance between the syringe tip and the collector was $10 \mathrm{~cm}$. An electrospinning voltage of $11-12 \mathrm{kV}$ was applied between the syringe tip and the aluminum foil.

The resultant electrospinning nanofibers were collected into a beaker which contained a mixed solution of ethanol and water by a glass rod on the collector. The as-prepared nanofibers were then washed with deionized water for three times. Finally, the appropriate amount of electrospinning nanofibers was allocated to the 12 -hole plate or 48 -hole plate and freeze dried overnight to obtain the fluffy nanofiber sponges (FNFSs).

2.3. Crosslinking. The chitosan was dissolved in $1.0 \%(v / v)$ acetic acid at a concentration of $0.050 \%, 0.075 \%, 0.100 \%$, and $0.125 \%(w / v)$. The as-prepared FNFSs were immersed into the chitosan solutions and then shocked slightly, followed by freeze-drying overnight to remove solvents. 


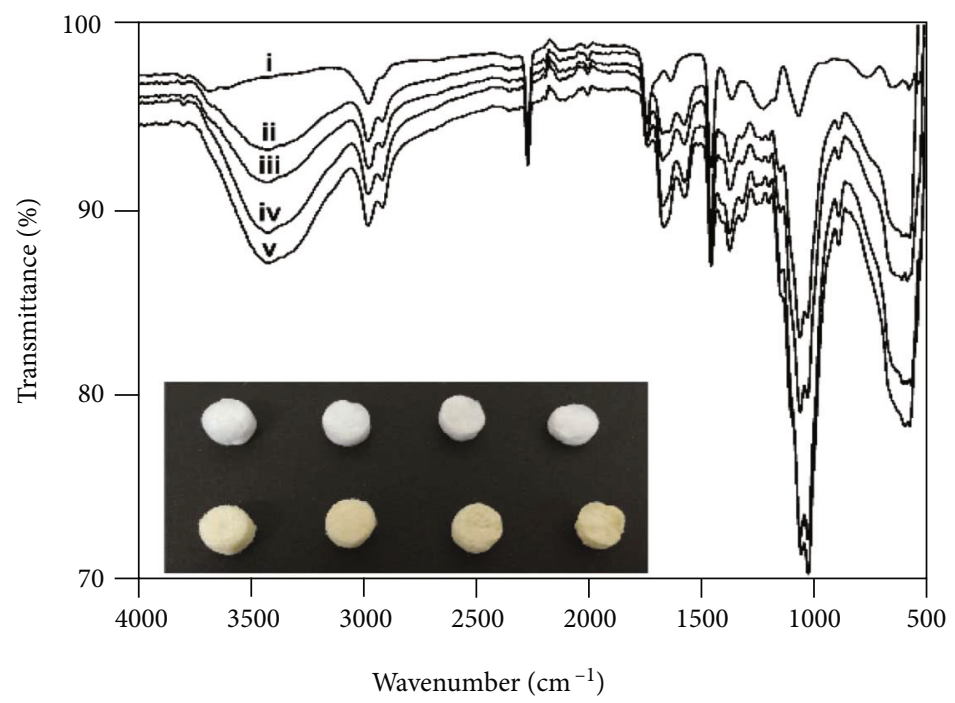

(a)

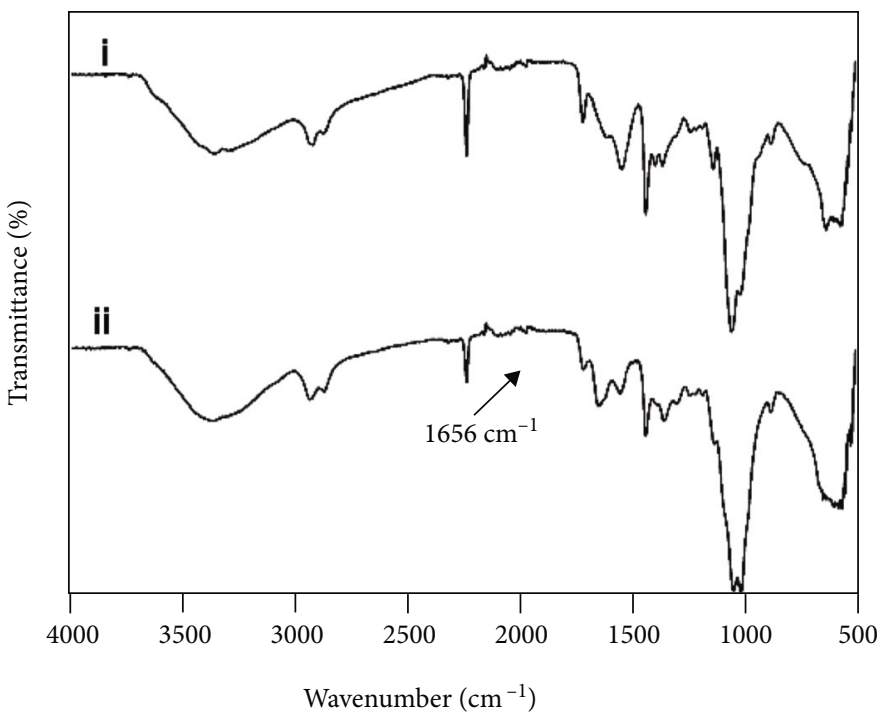

(b)

FIGURE 3: (a) FTIR spectra of PAN (i), $\mathrm{CNS}_{0.050}$ (ii), $\mathrm{CNS}_{0.075}$ (iii), $\mathrm{CNS}_{0.100}$ (iv), and $\mathrm{CNS}_{0.125}$ (v), with the inset showing the photographs of $\mathrm{CNS}_{0.050}, \mathrm{CNS}_{0.075}, \mathrm{CNS}_{0.100}$, and $\mathrm{CNS}_{0.125}$ from left to right (lower row) and their corresponding constructs before crosslinking (upper row). (b) FTIR spectra of $\mathrm{CNS}_{0.100}$ before (i) and after (ii) crosslinking.

A culture dish contained 25\% ( $v / v)$ glutaraldehyde solution was placed at the bottom of a dryer. The samples treated with chitosan were placed on the bracket above the culture dish at $40^{\circ} \mathrm{C}$ for $24 \mathrm{~h}$. The samples were then immersed in glycine solution $(0.1 \mathrm{M})$ to block the unreacted aldehyde group. Then, the samples were rinsed with PBS three times and dried to obtain the chitosan-coated nanofiber scaffolds (CNSs). The CNSs containing chitosan were named $\mathrm{CNS}_{0.050}, \mathrm{CNS}_{0.075}, \mathrm{CNS}_{0.100}$, and $\mathrm{CNS}_{0.125}$ according to their respective chitosan concentration.

2.4. Characterization. Infrared spectra of the PAN and CNS were obtained using a Nicolet iS10 FTIR instrument (Thermo Fisher Scientific, Waltham, MA) with wavenumber ranges from 400 to $4000 \mathrm{~cm}^{-1}$ by accumulating 16 scans at a resolution of $5 \mathrm{~cm}^{-1}$. The morphologies of three-dimensional FNFS, two-dimensional fibrous membrane obtained from the aluminum foil, and CNS with different concentrations of chitosan solution were observed by scanning electron microscopy (SEM). Prior to imaging, the specimens were sputtered with gold.

2.5. Cell Cytotoxicity Assays. The biocompatibility of the asprepared composites was evaluated by $3 \mathrm{~T} 3$ fibroblast cells. The 3T3 fibroblast cells were cultured on CNSs with different concentrations of chitosan solution, as the cells cultured on TCPs in the microplate were tested as the blank control.

TCP, $\mathrm{CNS}_{0.050}, \mathrm{CNS}_{0.075}, \mathrm{CNS}_{0.100}$, and $\mathrm{CNS}_{0.125}$ were examined in 5 groups. Before cell inoculation, all samples were immersed in $75 \%$ ethanol-water $(v / v)$ solution for 


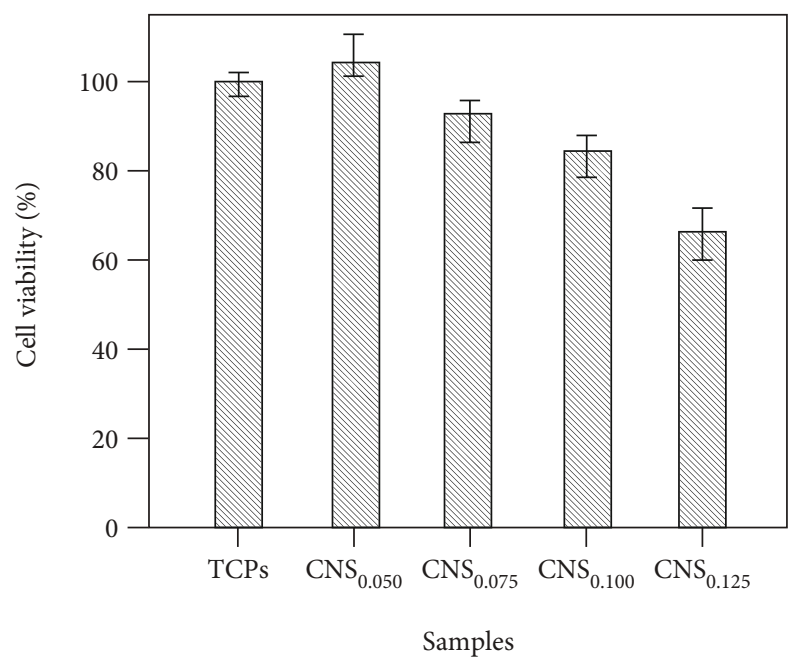

(a)
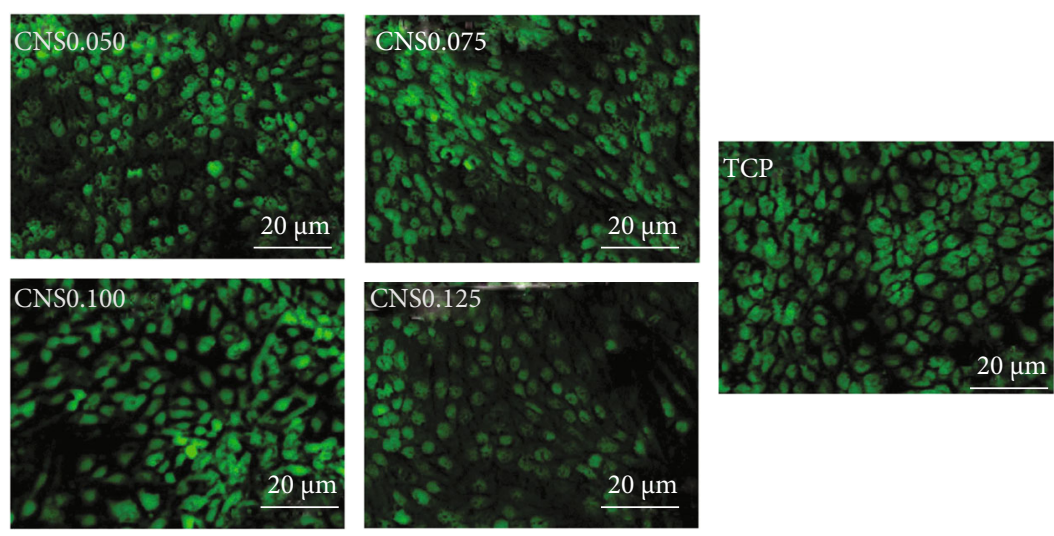

(b)

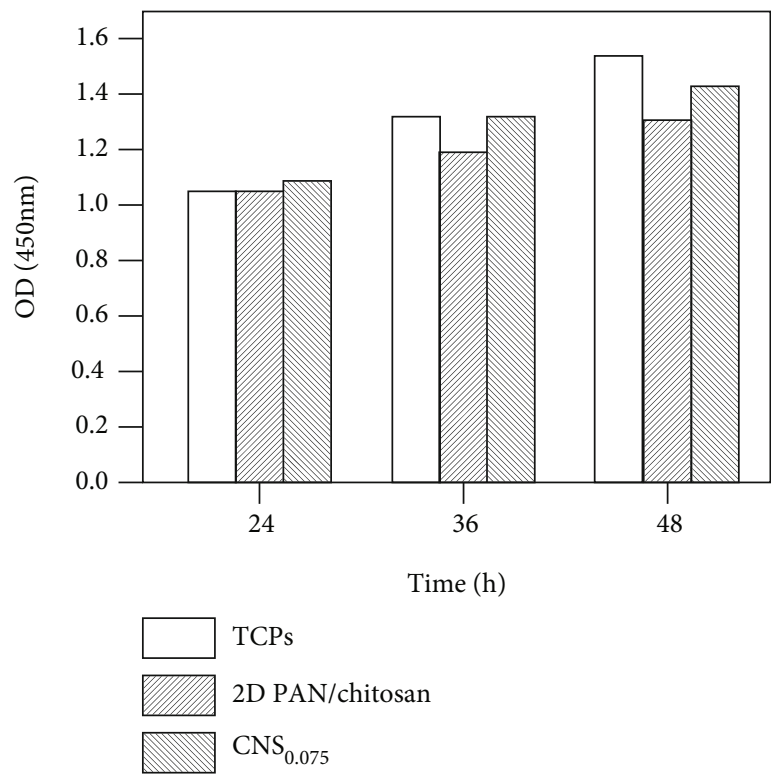

(c)

Figure 4: Continued. 

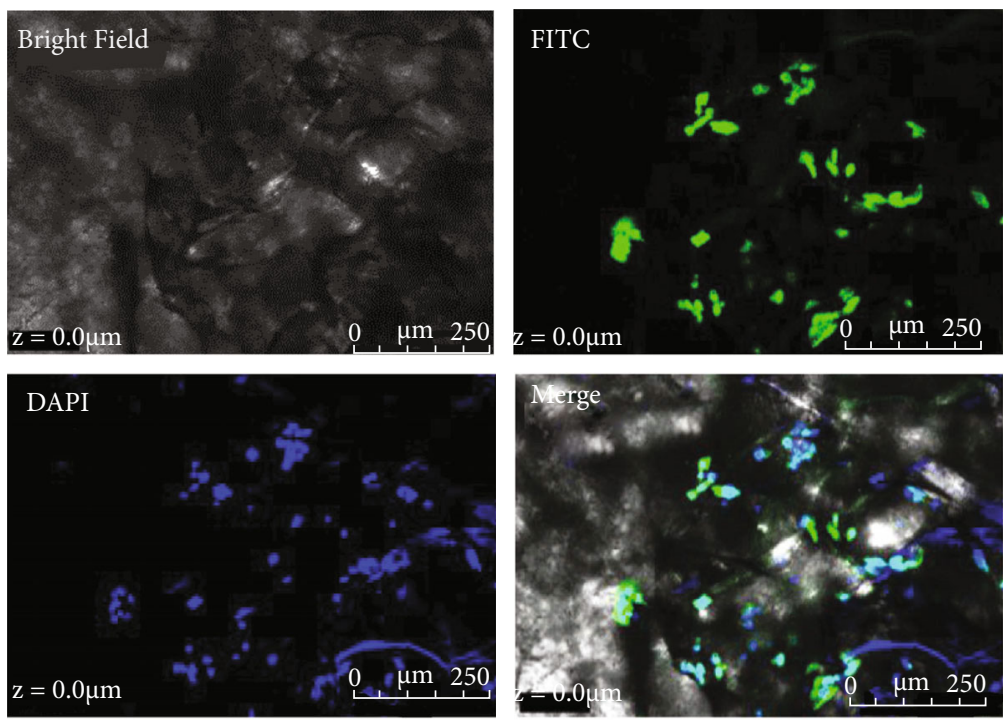

(d)

FIGURE 4: (a) Cell viability and (b) fluorescence images of $3 \mathrm{~T} 3$ fibroblast cells cultured on the TCPs, $\mathrm{CNS}_{0.050}, \mathrm{CNS}_{0.075}, \mathrm{CNS}_{0.100}$, and CNS ; (c) the proliferation of $3 \mathrm{~T} 3$ fibroblast cells cultured on the TCPs and $\mathrm{CNS}_{0.075}(24 \mathrm{~h}, 36 \mathrm{~h}, 48 \mathrm{~h})$; (d) fluorescence images of $3 \mathrm{~T} 3$ fibroblast cells cultured on $\mathrm{CNS}_{0.075}$.

$30 \mathrm{~min}$ and treated by ultraviolet radiation for another $30 \mathrm{~min}$ in the 24-well microplates. Thereafter, 3T3 fibroblast cells were seeded onto CNSs at a density of $1.5 \times 10^{4}$ cells per well. 3T3 fibroblast cells were cultured in medium (DMEM, 10\% $v / v$ fetal bovine serum and $1 \% v / v$ penicillin/streptomycin solution) at $37^{\circ} \mathrm{C}$ under $5 \% \mathrm{CO}_{2}$ in a cell culture incubator for 2 days.

The cell samples were washed with PBS for three times. Then, the cells were fixed with a $4 \%$ formaldehyde solution and stained with a Fluo-4 AM $(2.5 \mathrm{mM})$ solution. The standard MTT [22] assay was used to examine the viability of cells. Each sample was retested three times. The fluorescent images were taken by a fluorescence microscope (Olympus) configured with a Nuance CCD camera.

2.6. Cell Proliferation Assay. According to the same operation for the cell cytotoxicity assays, 3T3 fibroblast cells were cultured on the standard tissue culture plates (TCPs), two-dimensional PAN/chitosan cellulose membrane, and $\mathrm{CNS}_{0.075}$. The samples were taken for $24 \mathrm{~h}, 36 \mathrm{~h}$, and $48 \mathrm{~h}$. The wavelength of OD was measured at $450 \mathrm{~nm}$. The standard MTT assay was used to examine the cell proliferation. The cells were fixed with a $4 \%$ formaldehyde solution and stained with a $4^{\prime}, 6$-diamidino-2-phenylindole (DAPI) solution. Immunostaining the cytoskeleton of 3T3 fibroblast cells with FITC-labeled anti-Actin antibody was done as per the well-documented methods.

2.7. Antibacterial Experiment. Staphylococcus aureus (S. aureus) and Escherichia coli (E. coli) were selected and inoculated in $100 \mathrm{~mL}$ HS medium. The HS culture medium was composed of glucose 25 , yeast extract 5 , peptone 5 , citric acid monohydrate 1.2 , and $\mathrm{Na}_{2} \mathrm{HPO}_{4} 2.7\left(\mathrm{~g} \mathrm{~L}^{-1}\right)$. After inoculation, they were placed in the shaking table and shaken for 24 hours. $100 \mathrm{cfu} \mathrm{mL}^{-1}$ E. coli and S. aureus bacteria cells was grown in $50 \mathrm{~mL}$ liquid $\mathrm{LB}$ medium supplemented with $10 \mu \mathrm{g} \mathrm{mL}^{-1} \quad \mathrm{CNS}_{0}, \mathrm{CNS}_{0.050}, \mathrm{CNS}_{0.075}, \mathrm{CNS}_{0.100}$, and $\mathrm{CNS}_{0.125}$. The antibacterial activities of $\mathrm{CNS}_{0}, \mathrm{CNS}_{0.050}$, $\mathrm{CNS}_{0.075}, \mathrm{CNS}_{0.100}$, and $\mathrm{CNS}_{0.125}$ were determined by the shaking flask method. Growth rates and bacterial concentrations were determined by measuring the optical density (OD) of LB in broth medium at $600 \mathrm{~nm}$ every 6 hours.

\section{Results and Discussion}

3.1. Morphology of Fluffy Nanofiber Sponges (FNFSs). We successfully fabricated the fluffy nanofiber sponges (FNFSs) by the optimized electrospinning technique. The electrospinning process as shown in Scheme 1 was employed to fabricate the nanofibers in this study. $80 \%$ ethanol-water solution was used as the receiving device for electrospinning nanofibers to obtain the interconnected 3D (three-dimensional) structure. The SEM micrographs of three-dimensional FNFSs and twodimensional fibrous membrane obtained from the aluminum foil are shown in Figure 1. From it, we can see that the fiber spacing of the two-dimensional fibrous membrane was less than $5 \mu \mathrm{m}$ (Figure 1(a)), and fibers were accumulated and entangled mostly, with an average diameter of about $835 \mathrm{~nm}$ (Figure 1(b)). However, fiber spacing of the threedimensional FNFSs could reach from $15 \mu \mathrm{m}$ to $20 \mu \mathrm{m}$, and the fibers were obviously more dispersed from each other (Figure 1(c)) with an average diameter of about $808 \mathrm{~nm}$ (Figure 1(b)). The three-dimensional FNFS appeared as fibers with interconnected pores in the liquid environment (80\% ethanol), presenting properties that were not available in two-dimensional fiber membranes (aluminum foil), which provided good application prospect in adsorption materials, tissue engineering, and other fields. This fact suggests that the three-dimensional FNFSs received by liquid are more stable, and the diameter distribution is narrower. 


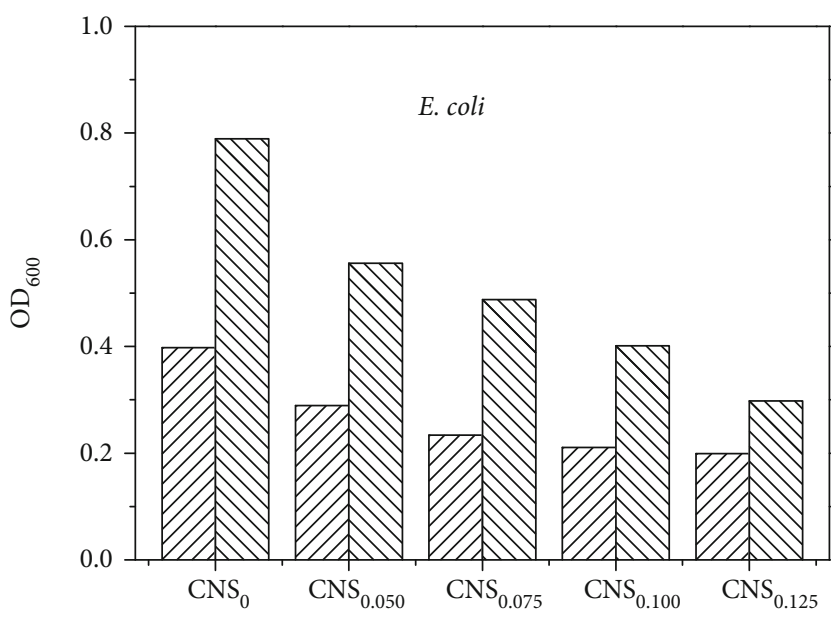

(a)

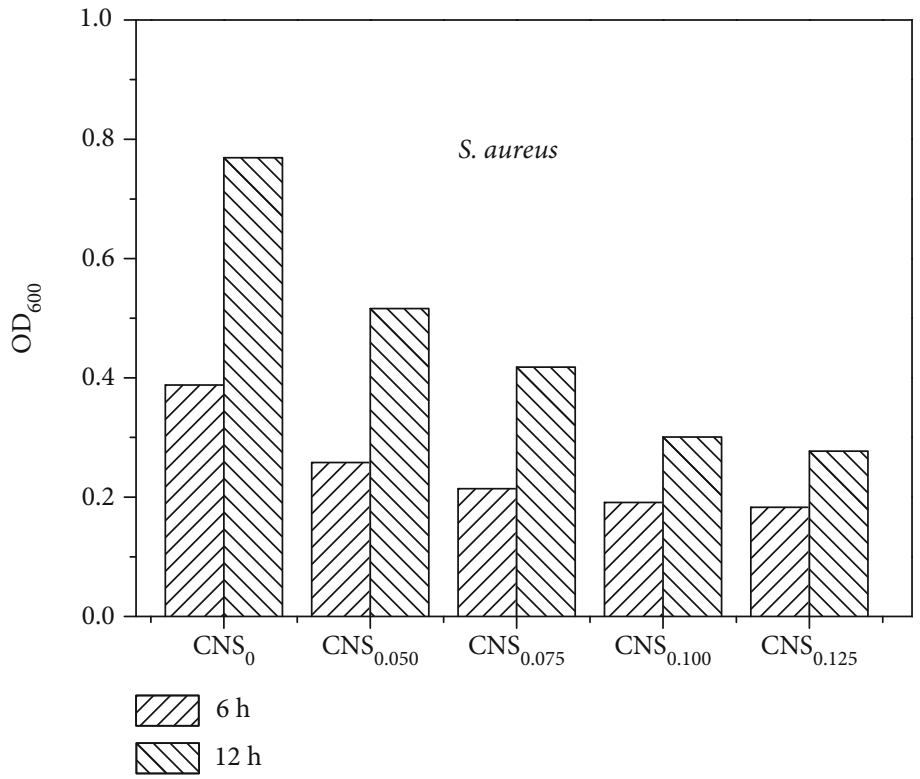

(b)

FIGURE 5: Bacteria suspension concentration $\left(\mathrm{OD}_{600}\right)$ of $\mathrm{CNS}_{0}, \mathrm{CNS}_{0.050}, \mathrm{CNS}_{0.075}, \mathrm{CNS}_{0.100}$, and $\mathrm{CNS}_{0.125}$ by E. coli (a) and S. aureus (b).

3.2. Characters of Chitosan-Coated Nanofiber Scaffolds (CNSs). The SEM micrographs of CNSs with different chitosan concentrations are shown in Figure 2. The chitosan looks like a layer of membranes at the surface of the CNSs, which do not affect the shape of the CNSs or the threedimensional mesh structure. With the increase of the concentration of chitosan from $0.050 \%$ to $0.125 \%(w / v)$, the chitosan nanofibers loaded on CNS increased obviously. On the one hand, the incorporation of chitosan with synthetic CNS scaffolds had shown vital advantages in improving scaffold biocompatibility, maintaining morphology and structural stability of three-dimensional scaffolds under low pressure. On the other hand, scaffolds with enough interconnected space were favorable for cell growth, migration, and proliferation.

Figure 3 exhibits the FT-IR spectra of PAN and CNSs. Three intense peaks at $2933 \mathrm{~cm}^{-1}, 2242 \mathrm{~cm}^{-1}$, and $1450 \mathrm{~cm}^{-1}$ belong to $\mathrm{C}-\mathrm{H}$ bands, $\mathrm{C} \equiv \mathrm{N}$ bands, and $-\mathrm{CH}_{2}$ bands, respec- tively $[23,24]$. The characteristic structures of PAN are observed both in the spectra of PAN and CNSs, showing the characteristic structures of PAN. There were three new peaks in the spectrum of CNSs appeared at $3368 \mathrm{~cm}^{-1}$, $2870 \mathrm{~cm}^{-1}$, and $1559 \mathrm{~cm}^{-1}$, which could, respectively, be due to the telescopic vibration of the $\mathrm{O}-\mathrm{H}$, the telescopic vibration of the $\mathrm{C}-\mathrm{H}$, and the bending vibration of the $\mathrm{N}-\mathrm{H}$ in molecular chitosan [25]. Taking these results into consideration, it was clear that the combined electrospinning scaffolds (CNSs) contained both PAN and chitosan. Figure 3(b) displays the FT-IR spectra of $\mathrm{CNS}_{0.01}$ before (i) and after (ii) crosslinking. After crosslinking, the characteristic $\mathrm{N}=\mathrm{C}$ telescopic vibration peak at $1656 \mathrm{~cm}^{-1}$ becomes stronger and the characteristic $\mathrm{N}-\mathrm{H}$ bending vibration absorption peak becomes weaker. Combining the photos of CNS with the photos of CNS after crosslinking, it can be found that due to the formation of $\mathrm{N}=\mathrm{C}$ bands, $\mathrm{CNS}$ becomes slightly yellow. 
3.3. Cytotoxicity and Cell Proliferation of CNSs. The $3 \mathrm{~T} 3$ fibroblast cells as model cells were cultured on CNSs with different concentrations of chitosan solution for 2 days. The cytotoxicity of different samples was evaluated by MTT cell viability assay. As shown in Figure 4(a), 3T3 fibroblast cells maintained nearly $104 \%$ and $92 \%$ of cell viability on the substance of $\mathrm{CNS}_{0.050}$ and $\mathrm{CNS}_{0.075}$, respectively, but only $87 \%$ and $67 \%$ of $\mathrm{CNS}_{0.100}$ and $\mathrm{CNS}_{0.125}$, respectively. The blank control experiments were included using the standard tissue culture plates (TCPs) for comparison with normalization. It suggests that both $\mathrm{CNS}_{0.050}$ and $\mathrm{CNS}_{0.075}$ exhibit very low cytotoxicity for 3T3 fibroblast cells. 3T3 cells were imaged with a fluorescent microscope after they were cultured on TCPs and CNSs with different concentrations of chitosan for 2 days. As shown in Figure 4(b), the number of cells cultured on $\mathrm{CNS}_{0.050}$ and $\mathrm{CNS}_{0.075}$ are similar to that on TCPs. It also confirmed the low cytotoxicity of $\mathrm{CNS}_{0.050}$ and $\mathrm{CNS}_{0.075}$ for 3T3 fibroblast cells. Meanwhile, it was obvious that the cells growing on smooth TCP were better distributed because of the freedom of cell migration, while the growth of the cells cultured on CNS presented local directivity, indicating that the cells basically grew towards the direction of the fibers. These results suggested that $\mathrm{CNS}_{0.050}$ and $\mathrm{CNS}_{0.075}$ could provide good biocompatibility and wellcontrolled microenvironments for the growth and proliferation of 3T3 fibroblast cells.

In order to investigate the proliferation of $3 \mathrm{~T} 3$ fibroblast cells cultured on $\mathrm{CNS}_{0.075}$, the control experiments were included using the standard tissue culture plates (TCPs) and the two-dimensional PAN/chitosan cellulose membrane for comparison with normalization. Figure 4(c) shows the proliferation of cells which were cultured for $24 \mathrm{~h}, 36 \mathrm{~h}$, and $48 \mathrm{~h}$. After $24 \mathrm{~h}$ in culture, the proliferation of cells on these three materials was almost the same. After $36 \mathrm{~h}$ in culture, the proliferation of cells on the two-dimensional PAN/chitosan cellulose membrane and $\mathrm{CNS}_{0.075}$ began to be lower than that of TCPs. After $48 \mathrm{~h}$ in culture, the proliferation of cells on the CNS was about $90 \%$ of TCPs but higher than that on the two-dimensional PAN/chitosan membrane. Taken together, these results suggest that CNS three-dimensional scaffolds can provide good biocompatibility and well-controlled microenvironments for cell growth and proliferation, which can be applied to wound healing in the future.

From Figure 4(d), we can see that the 3T3 fibroblast cell growth forms between the multilayered structures of the materials. The cellular skeleton structure was immunostained with the primary antibody (Rabbit, antitubulin antibody) and the secondary antibody (Goat anti-Rabbit IgG, FITC-labelled by Phalloidin-Oregon). Cell nuclei were stained by Hoechst (DAPI). The images displayed that $3 \mathrm{~T} 3$ fibroblast cells grew very well in the $3 \mathrm{D}$ space of $\mathrm{CNS}_{0.075}$ substrates. This preliminary experiment suggests that the 3D-CNS composite has a good biocompatibility in terms of cell culture, resulting in good 3D microenvironments for cell culture.

3.4. Antibacterial Performance. The antibacterial performance of the $\mathrm{CNS}_{0}, \mathrm{CNS}_{0.050}, \mathrm{CNS}_{0.075}, \mathrm{CNS}_{0.100}$, and $\mathrm{CNS}_{0.125}$ nanofibers was investigated by both Gram- negative bacterial strains, E. coli, and Gram-positive bacterial strains, S. aureus [26]. As shown in Figure 5, the bacterial $\mathrm{OD}_{600}$ values of $\mathrm{CNS}_{0.050}, \mathrm{CNS}_{0.075}, \mathrm{CNS}_{0.100}$, and $\mathrm{CNS}_{0.125}$ were lower than that of $\mathrm{CNS}_{0}$ (the control group) in the experiments of relatively short incubation time $(6 \mathrm{~h}, 12 \mathrm{~h})$, on both $E$. coli and S. aureus, due to the antibacterial properties of chitosan [27]. Furthermore, the $\mathrm{OD}_{600}$ percentage of $S$. aureus bacterial suspension with CNS after $12 \mathrm{~h}$ cultivation is lower than that of E. coli, which indicated that CNS has better antibacterial properties for Gram-positive bacteria than Gram-negative bacteria [28].

\section{Conclusions}

Here, the fluffy nanofiber sponge was successfully prepared by controlling the process parameters of liquid-assisted electrospinning. Compared with the traditional electrospinning technology, the nanofiber spacing can reach to $15-20 \mu \mathrm{m}$, forming an interconnected pore structure. Then, chitosan was loaded on the prepared nanofibers and crosslinked. The composite scaffolds were used as three-dimensional scaffolds for 3T3 fibroblasts in vitro, and a series of cell experiments and in vitro antibacterial experiments were carried out. The results show that these composite threedimensional scaffolds have low cytotoxicity, providing a good biological microenvironment for cell adhesion, migration, and proliferation, and are conducive to self-repair of damaged tissue in the wound. At the same time, these three-dimensional composite scaffolds have good antibacterial properties due to chitosan loading, which can effectively prevent wound infection in practical application, where traditional medical dressings cannot achieve. In addition, chitosan itself has excellent loading capacity, which is often used to make a carrier. The addition of chitosan provides great help for loading new drugs into wound dressings and simplifies the process. In the future, we believe that the excellent properties of this kind of CNS material will be used in biology, adsorption materials, tissue engineering, and other fields.

\section{Data Availability}

Data are available on request.

\section{Conflicts of Interest}

The authors declare no conflict of interest.

\section{Acknowledgments}

This work was supported by the National Natural Science Foundation of China (Grants 51803092 and 51873087), General Research Project of Jiangsu Provincial Health Commission (H2018058), Natural Science Foundation of Jiangsu Province of China (BK20180490), Fundamental Research Funds for the Central Universities (Grant 30920130121001), and a project funded by the Priority Academic Program Development of Jiangsu Higher Education Institutions (PAPD, China). 


\section{References}

[1] N. Bhardwaj and S. C. Kundu, "Electrospinning: a fascinating fiber fabrication technique," Biotechnology Advances, vol. 28, no. 3, pp. 325-347, 2010.

[2] S. Agarwal, A. Greiner, and J. H. Wendorff, "Functional materials by electrospinning of polymers," Progress in Polymer Science, vol. 38, no. 6, pp. 963-991, 2013.

[3] B. Sun, Y. Z. Long, H. D. Zhang et al., "Advances in threedimensional nanofibrous macrostructures via electrospinning," Progress in Polymer Science, vol. 39, no. 5, pp. 862890, 2014.

[4] W. Teo, S. Liao, C. Chan, and S. Ramakrishna, "Remodeling of three-dimensional hierarchically organized nanofibrous assemblies," Current Nanoscience, vol. 4, no. 4, pp. 361-369, 2008.

[5] Q. P. Pham, U. Sharma, and A. G. Mikos, "Electrospun poly ( $\varepsilon$ caprolactone) microfiber and multilayer nanofiber/microfiber scaffolds: characterization of scaffolds and measurement of cellular infiltration," Biomacromolecules, vol. 7, no. 10, pp. 2796-2805, 2006.

[6] S. Soliman, S. Pagliari, A. Rinaldi et al., "Multiscale threedimensional scaffolds for soft tissue engineering via multimodal electrospinning," Acta Biomaterialia, vol. 6, no. 4, pp. 1227-1237, 2010

[7] R. Gentsch, B. Boysen, A. Lankenau, and H. G. Börner, "Single-step electrospinning of bimodal fiber meshes for ease of cellular infiltration," Macromolecular Rapid Communications, vol. 31, no. 1, pp. 59-64, 2010.

[8] M. R. Badrossamay, H. A. McIlwee, J. A. Goss, and K. K. Parker, "Nanofiber assembly by rotary jet-spinning," Nano Letters, vol. 10, no. 6, pp. 2257-2261, 2010.

[9] L. Dimesso, C. Spanheimer, W. Jaegermann, Y. Zhang, and A. Yarin, " $\mathrm{LiFePO} \mathrm{P}_{4}-3 \mathrm{D}$ carbon nanofiber composites as cathode materials for Li-ions batteries," Journal of Applied Physics, vol. 111, no. 6, article 064307, 2012.

[10] R. Bagherzadeh, M. Latifi, S. S. Najar, M. Gorji, and L. Kong, "Transport properties of multi-layer fabric based on electrospun nanofiber mats as a breathable barrier textile material," Textile Research Journal, vol. 82, no. 1, pp. 70-76, 2012.

[11] T. G. Kim, H. J. Chung, and T. G. Park, "Macroporous and nanofibrous hyaluronic acid/collagen hybrid scaffold fabricated by concurrent electrospinning and deposition/leaching of salt particles," Acta Biomaterialia, vol. 4, no. 6, pp. 16111619, 2008.

[12] Y. Chen, M. Shafiq, M. Liu, M. Yosrey, and X. Mo, "Advanced fabrication for electrospun three-dimensional nanofiber aerogels and scaffolds," Bioactive Materials, vol. 5, no. 4, pp. 963979, 2020.

[13] A. Ti, A. Swh, and B. Mda, "Fabrication of aligned $\mathrm{PAN} / \mathrm{TiO}_{2}$ fiber using electric electrospinning (EES)," Materials Today: Proceedings, vol. 13, Part 1, pp. 211-216, 2019.

[14] M. Shin, H. Yoshimoto, and J. P. Vacanti, "In vivo bone tissue engineering using mesenchymal stem cells on a novel electrospun nanofibrous scaffold," Tissue Engineering, vol. 10, no. 12, pp. 33-41, 2004.

[15] W. Friess, "Collagen - biomaterial for drug delivery ${ }^{1}$," European Journal of Pharmaceutics and Biopharmaceutics, vol. 45, no. 2, pp. 113-136, 1998.

[16] M. Vong, F. Sanchez, A. Keirouz, W. Nuansing, and N. Radacsi, "Ultrafast fabrication of nanofiber-based 3D mac- rostructures by 3D electrospinning," Materials \& Design, vol. 208, article 109916, 2021.

[17] S. J. Kim, D. H. Jang, W. H. Park, and B.-M. Min, "Fabrication and characterization of 3-dimensional PLGA nanofiber/ microfiber composite scaffolds," Polymer, vol. 51, no. 6, pp. 1320-1327, 2010.

[18] S. Wang, Y. Zhang, H. Wang, G. Yin, and Z. Dong, "Fabrication and properties of the electrospun polylactide/silk fibroin-gelatin composite tubular scaffold," Biomacromolecules, vol. 10, no. 8, pp. 2240-2244, 2009.

[19] I. K. Shim, M. R. Jung, K. H. Kim et al., "Novel threedimensional scaffolds of poly(L-lactic acid) microfibers using electrospinning and mechanical expansion: fabrication and bone regeneration," Journal of Biomedical Materials Research Part B: Applied Biomaterials, vol. 95B, no. 1, pp. 150-160, 2010.

[20] R. Krishnan, R. Rajeswari, J. Venugopal et al., "Polysaccharide nanofibrous scaffolds as a model for in vitro skin tissue regeneration," Journal of Materials Science: Materials in Medicine, vol. 23, no. 6, pp. 1511-1519, 2012.

[21] Y. Zhi, S. Zhicheng, N. Xin, G. Kaijin, and G. Yan, “A smart scaffold composed of three-dimensional printing and electrospinning techniques and its application in rat abdominal wall defects," Stem Cell Research Therapy, vol. 11, no. 1, p. 533, 2020.

[22] M. Hanelt, M. Gareis, and B. Kollarczik, "Cytotoxicity of mycotoxins evaluated by the MTT-cell culture assay," Mycopathologia, vol. 128, no. 3, pp. 167-174, 1994.

[23] S. S. Kim and J. Lee, "Antibacterial activity of polyacrylonitrilechitosan electrospun nanofibers," Carbohydrate Polymers, vol. 102, pp. 231-237, 2014.

[24] E. A. El-Hefian, M. M. Nasef, and A. H. Yahaya, "Preparation and characterization of chitosan/agar blended films: part 1 . Chemical structure and morphology," Journal of Chemistry, vol. 9, no. 3, 1439 pages, 2012.

[25] M. M. Beppu, R. S. Vieira, C. G. Aimoli, and C. C. Santana, "Crosslinking of chitosan membranes using glutaraldehyde: effect on ion permeability and water absorption," Journal of Membrane Science, vol. 301, no. 1-2, pp. 126-130, 2007.

[26] L. Yu, Y. Zhang, B. Zhang, and J. Liu, "Enhanced antibacterial activity of silver nanoparticles/halloysite nanotubes/graphene nanocomposites with sandwich-like structure," Scientific Reports, vol. 4, no. 1, p. 4551, 2015.

[27] T. Jin, T. Liu, E. Lam, and A. H. Moores, "Chitin and chitosan on the nanoscale," Nanoscale Horizons, vol. 6, no. 7, pp. 505542, 2021.

[28] R. Lian, J. Cao, X. Jiang, and A. V. Rogachev, "Physicochemical, antibacterial properties and cytocompatibility of starch/ chitosan films incorporated with zinc oxide nanoparticles," Communications, vol. 27, p. 102265, 2021. 